\title{
ON SOME CENTER-LIKE SUBSETS OF GROUPS
}

\section{ABRAHAM A. KLEIN and HOWARD E. BELL}

\author{
Received 1 March 2003
}

We study the properties of certain center-like subsets of groups, which are obtained by localizing setwise commutativity conditions.

2000 Mathematics Subject Classification: 20F99.

1. Introduction. Let $G$ denote a group with center $Z=Z(G)$; and for each subset $A$ of $G$, denote by $C(A)$ the centralizer of $A$ in $G$. In this paper, we define several "center-like" subsets of $G$, investigate their properties and establish conditions for them to coincide with $Z$.

2. The Freiman center. In [1], Freiman proved the following theorem.

THEOREM 2.1. Let $G$ be a group with the property that for each $a, b \in G$, $\left|\{a, b\}^{2}\right|=\left|\left\{a^{2}, a b, b a, b^{2}\right\}\right| \leq 3$. Then either $G$ is abelian or $G=Q \times E$, where $Q$ is the quaternion group and $E$ is an elementary abelian 2-group.

Motivated by this theorem, we define the Freiman center $F_{r}(G)$ to be the set

$$
\left\{a \in G||\{a, x\}^{2} \mid \leq 3 \forall x \in G\right\} .
$$

It is obvious that $Z(G) \subseteq F_{r}(G)$ for all groups $G$, and $Q$ is a group such that $Z(Q) \neq F_{r}(Q)=Q$. Initially, it is not evident that $F_{r}(G)$ has any particular structure in general; however, if we can show that it is a subgroup of $G$, then Theorem 2.1 gives information about its structure.

LEMMA 2.2. Let $G$ be a group for which $F_{r}(G) \neq Z$. If $a \in F_{r}(G) \backslash Z$ and $b \notin C(a)$, then $a^{2}=b^{2}=(a b)^{2}=(b a)^{2}, b a b=a$, and $a b a=b$. Moreover, $a^{4}=b^{4}=1 \neq a^{2}$.

Proof. The first statement is obvious from the definition of $F_{r}(G)$. If $z \in Z$, or more generally if $z \in C(a, b)$, then $a \notin C(b z)$; hence $a^{2}=(b z)^{2}=b^{2} z^{2}=b^{2}$; and thus $z^{2}=1$. Since $a^{2}=b^{2}$ for all $b \notin C(a), a^{2} \in Z$ and therefore $a^{4}=1$. Since $a b a=b, a^{2}=1$ would imply that $a b=b a$, contrary to our hypothesis that $b \notin C(a)$; therefore $a^{2} \neq 1$. 
LEMMA 2.3. Let $G$ be a group in which $F_{r}(G) \neq Z$. Then

(i) $Z=\left\{z \in G \mid z^{2}=1\right\}$;

(ii) if $a \in F_{r}(G) \backslash Z$ and $b \notin C(a), Z=C(a, b)$.

Proof. (i) Let $Z_{1}=\left\{z \in G \mid z^{2}=1\right\}$. We noted in the proof of Lemma 2.2 that $Z \subseteq Z_{1}$. To show that $Z_{1} \subseteq Z$, let $a \in F_{r}(G) \backslash Z, b \notin C(a)$, and $z \in Z_{1}$. Then by Lemma 2.2, $z \in C(a)$ and hence $b z \notin C(a)$. Therefore, $b^{2}=a^{2}=(b z)^{2}$ so that $b=z b z$ and $b z=z b$. We have shown that $z$ centralizes the complement of the proper subgroup $C(a)$, hence $z \in Z$.

(ii) Obviously, $Z \subseteq C(a, b)$. It was noted in the proof of Lemma 2.2 that $C(a, b) \subseteq Z_{1}$, so by (i), $C(a, b) \subseteq Z$.

We can now show that $F_{r}(G)$ is a subgroup of $G$.

THEOREM 2.4. If $G$ is any group, $F_{r}(G)$ is a characteristic subgroup of $G$. Moreover, if $F_{r}(G) \neq Z$, then $F_{r}(G)$ is of exponent 4 .

Proof. Since $a^{-1}=a^{3}$ for all $a \in F_{r}(G) \backslash Z$, to show that $F_{r}(G)$ is a subgroup, we need only to establish closure under the group operation. Let $a, b \in$ $F_{r}(G)$. Of course, if $a, b \in Z$, there is nothing to prove. Now consider $a \in$ $F_{r}(G) \backslash Z$ and $b \in Z$. If $x \in C(a)$, then $x a b=a b x$; and if $x \notin C(a)$, Lemmas 2.2 and 2.3(i) give $(a b)^{2}=a^{2} b^{2}=a^{2}=x^{2}$. In either event, $\left|\{a b, x\}^{2}\right| \leq 3$.

We are left with the case $a, b \in F_{r}(G) \backslash Z$. If $x \in C(a) \cap C(b)$, then $x a b=a b x$; so we assume $x \notin C(a)$, in which case $a^{2}=x^{2}$. If $b \notin C(a)$, it follows by Lemma 2.2 that $(a b)^{2}=a^{2}$, so $(a b)^{2}=x^{2}$. If $b \in C(a)$, Lemma 2.3(ii) gives $x \notin C(b)$; and by Lemma 2.2, we have $x^{2}=b^{2}=a^{2}$ and $a x a=x=b x b$. Thus $a b x a b=b a x a b=x$; and since $(a b)^{2}=a^{2} b^{2}=a^{4}=1$, we have $a b x=x a b$.

We have now shown that $F_{r}(G)$ is a subgroup. It is obviously invariant under automorphisms; and if it is different from $Z$, it is of exponent 4 by Lemmas 2.2 and 2.3(i).

The next result follows at once from Lemma 2.2, which implies that $a \in$ $F_{r}(G) \backslash Z$ and $b \notin C(a)$ generate a subgroup isomorphic to $Q$.

THEOREM 2.5. If $G$ is any group which does not contain $Q$ as a subgroup, then $F_{r}(G)=Z$.

In order to obtain our next major result, we need two additional lemmas.

LEMMA 2.6. Let $G$ be a group for which $F_{r}(G) \neq Z$. If $a \in F_{r}(G) \backslash Z$ and $b \notin C(a)$, then $G=C(a) \cup b C(a)$.

Proof. Note that $b a b^{-1}=b a b^{3}=(b a b) b^{2}=a^{3}$; and for any $c \notin C(a)$, $c a c^{-1}=a^{3}$, and hence $b a b^{-1}=c a c^{-1}$. Thus $b^{-1} c \in C(a)$ and $c \in b C(a)$.

LEMMA 2.7. Let $G$ be a group such that $F_{r}(G)$ is not commutative. If $a \in$ $F_{r}(G)$ is an element for which there exists $b \in F_{r}(G)$ such that $a b \neq b a$, then $C(a)=Z \cup a Z$. 
Proof. For $a, b \in F_{r}(G)$ with $a b \neq b a$, we have $a^{2}=b^{2}$. Moreover, if $c \in$ $C(a) \backslash Z$, then by Lemma 2.3(ii), we get $c \notin C(b)$; hence $b^{2}=c^{2}$. Thus $\left(a^{-1} c\right)^{2}=$ $a^{-2} c^{2}=b^{-2} b^{2}=1$, hence $a^{-1} c \in Z$ and $c \in a Z$.

We can now prove our main theorem on $F_{r}(G)$.

THEOREM 2.8. For any group $G$, one of the following is true:

(i) $F_{r}(G)=Z$;

(ii) $F_{r}(G)=G$;

(iii) $F_{r}(G)=Z \cup a Z$ for any $a \in F_{r}(G) \backslash Z$.

Proof. Assume that $F_{r}(G) \neq Z$. If $F_{r}(G)$ is not commutative, then by Lemmas 2.6 and 2.7, there exist $a, b \in F_{r}(G) \backslash Z$ such that $G=C(a) \cup b C(a)=$ $Z \cup a Z \cup b Z \cup b a Z$; hence $G=F_{r}(G)$.

Finally, we consider the case of $F_{r}(G)$ commutative but not central. Note that for any $a, b \in F_{r}(G) \backslash Z$, we have $C(a)=C(b)$, for $x \in C(a) \backslash C(b)$ would imply $a \in C(b, x)=Z$. Thus, for any $a, b \in F_{r}(G) \backslash Z$ and any $x \in G$, either $x \in C\left(a^{-1} b\right)$ or $x \notin C(a)=C(b)$; and in the latter case $a^{2}=x^{2}=b^{2}$ so that $\left(a^{-1} b\right)^{2}=a^{-2} b^{2}=1$, and hence $a^{-1} b \in Z$. Therefore, $G=C\left(a^{-1} b\right), a^{-1} b \in Z$, and $b \in a Z$.

It is clear from Theorems 2.1 and 2.5 that (i) and (ii) can actually occur. Seeking a finite group $G$ for which (iii) holds, we note that $|G|$ must be divisible by 8 (by Theorem 2.5); and we examine the non-abelian groups of order 16. By Lemmas 2.2 and 2.6, there must be at least 9 elements with the same square. In fact, the only non-abelian group of order 16 with this property is the dicyclic group $Q_{8}$-type 16/14 in [2]; and this group does satisfy (iii).

3. The strong Freiman center. It is amusing to consider what happens if we tighten the definition of Freiman center. We define the strong Freiman center $\hat{F}_{r}(G)$ to be $\left\{a \in G||\{a, x\}^{2} \mid \leq 2\right.$ for all $\left.x \in G\right\}$.

It is easy to find groups $G$ for which $\widehat{F}_{r}(G)$ is empty. On the other hand, if $G$ is an elementary 2-group, then $\hat{F}_{r}(G)=G$. The next theorem states that there are no other nontrivial $G$ for which $\widehat{F}_{r}(G) \neq \varnothing$.

THEOREM 3.1. If $G$ is any group, one of the following holds:

(i) $\hat{F}_{r}(G)=\phi$;

(ii) $G=\{1\}$;

(iii) $G$ is an elementary 2-group.

Proof. Note first that if $G$ is any non-abelian group with $a \notin Z$ and $b \notin$ $C(a)$, then $\left|\{a, b\}^{2}\right| \geq 3$; therefore $\hat{F}_{r}(G) \subseteq Z$ for all groups $G$. Assume now that $\hat{F}_{r}(G) \neq \phi$ and $a \in \hat{F}_{r}(G)$. If $|G|>1$, then for any $b \neq a,\{a, b\}^{2}=\left\{a^{2}, b^{2}, a b\right\}$; hence $a^{2}=b^{2}$. Thus $x^{2}=1$ for all $x \in G$. 
4. The Neumann centers. The following definitions resulted from a conversation between the second author and B. H. Neumann.

For a fixed positive integer $n$, let

$$
T_{n}(G)=\{x \in G \mid x S=S x \text { for every } n \text {-subset } S \text { of } G\},
$$

and let

$$
T_{\infty}(G)=\{x \in G \mid x S=S x \text { for every infinite subset } S \text { of } G\} .
$$

It is clear that $Z=T_{1}(G) \subseteq T_{n}(G) \subseteq T_{n+1}(G) \subseteq T_{\infty}(G)$ for all $n$. It is easy to see that $T_{\infty}(G)$ and all $T_{n}(G)$ are characteristic subgroups of $G$. Moreover, if $|G| \leq n$, then $T_{n}(G)=T_{\infty}(G)=G$.

Our principal theorem in this section states that there are only two possibilities for $T_{n}(G)$ or $T_{\infty}(G)$ : either $Z$ or $G$.

THEOREM 4.1. If $G$ is a group with $|G|>n$, then $T_{n}(G)=Z$; and if $G$ is any infinite group, $T_{\infty}(G)=Z$.

Proof. We prove the first assertion; the proof of the second is essentially the same. Let $|G|>n$, and suppose $x \in G \backslash Z$. There exists $y \in G$ such that $x y \neq y x$; and there exists a unique $w \in G$ such that $x y=w x$. Taking $S$ to be an $n$-subset containing $y$ but not $w$, we have $x y \in x S \backslash S x$, hence $x \notin T_{n}(G)$. Thus, $T_{n}(G) \subseteq Z$.

COROLLARY 4.2. For any group $G$ and any $n \leq 5, T_{n}(G)=Z$.

REMARK 4.3. We can, in fact, define $T_{\alpha}(G)$ for any cardinal number $\alpha-$ as $\{x \in G \mid x S=S x$ for all subsets $S$ of cardinality $\alpha\}$. Then the extension of Theorem 4.1 states that $T_{\alpha}(G)=Z$ if $\alpha$ is finite and $|G|>\alpha$, or if $\alpha$ is infinite and $|G| \geq \alpha$, and that in all other cases $T_{\alpha}(G)=G$.

ACKNOWLEDGMENT. This work was supported by the Natural Sciences and Engineering Research Council of Canada, Grant 3961.

\section{REFERENCES}

[1] G. A. Freiman, On two- and three-element subsets of groups, Aequationes Math. 22 (1981), no. 2-3, 140-152.

[2] A. D. Thomas and G. V. Wood, Group Tables, Shiva Mathematics Series, vol. 2, Shiva Publishing, Nantwich, 1980.

Abraham A. Klein: Sackler Faculty of Exact Sciences, School of Mathematical Sciences, Tel Aviv University, Tel Aviv 69978, Israel

E-mail address: aak1ein@post.tau.ac. 11

Howard E. Bell: Department of Mathematics, Brock University, St. Catharines, Ontario, Canada L2S 3A1

E-mail address: hbe11@spartan.ac.brocku.ca 


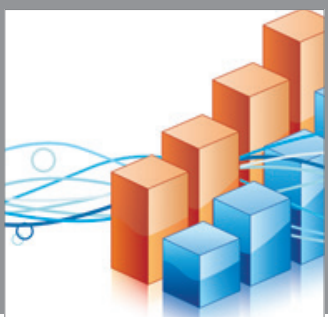

Advances in

Operations Research

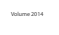

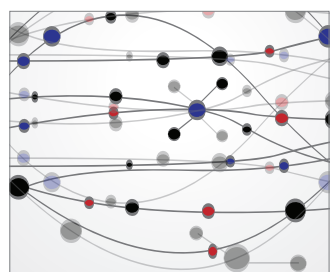

\section{The Scientific} World Journal
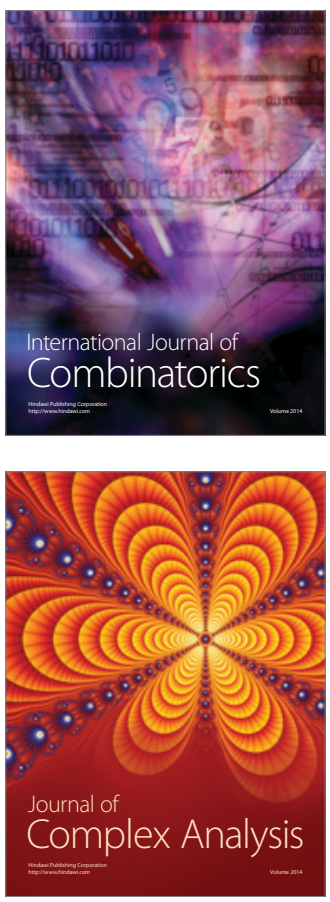

International Journal of

Mathematics and

Mathematical

Sciences
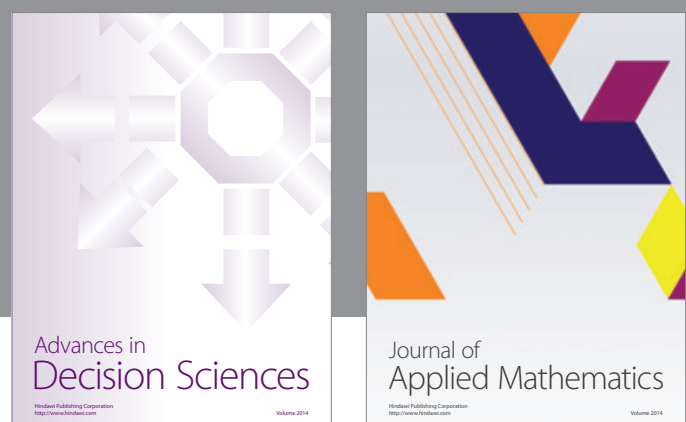

Journal of

Applied Mathematics
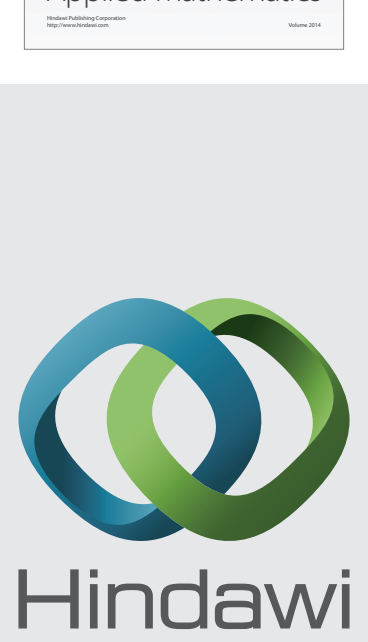

Submit your manuscripts at http://www.hindawi.com
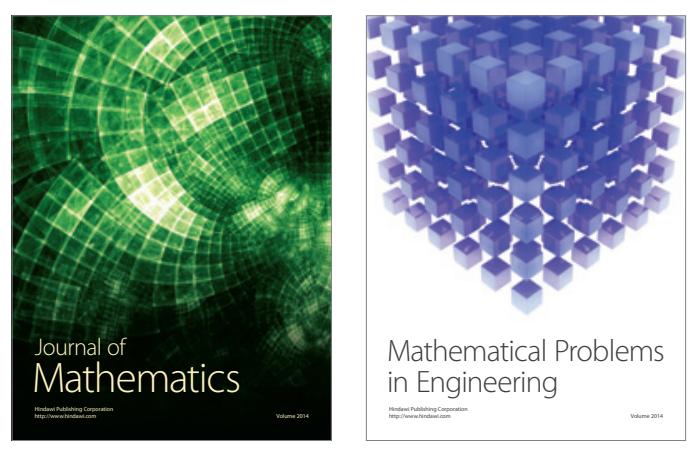

Mathematical Problems in Engineering
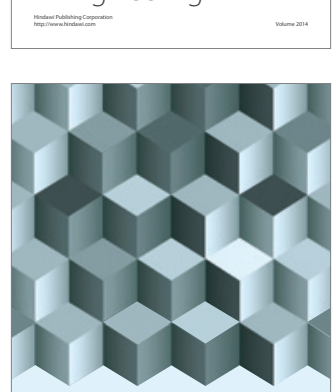

Journal of

Function Spaces
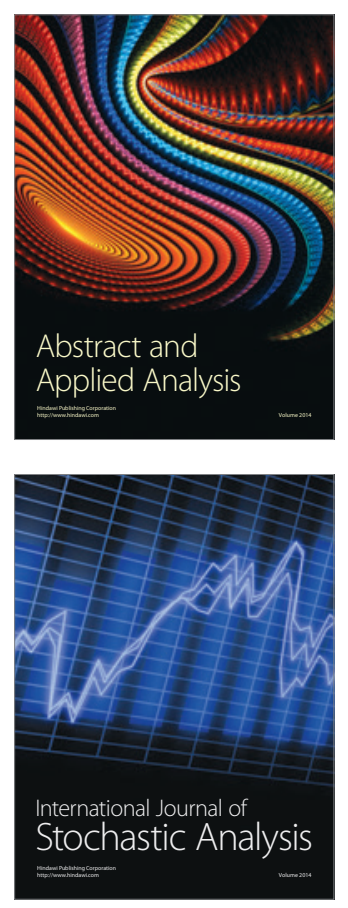

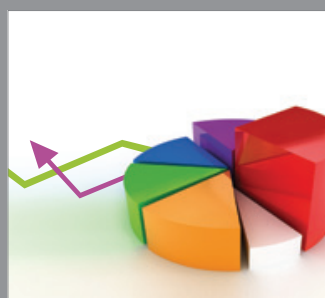

ournal of

Probability and Statistics

Promensencen
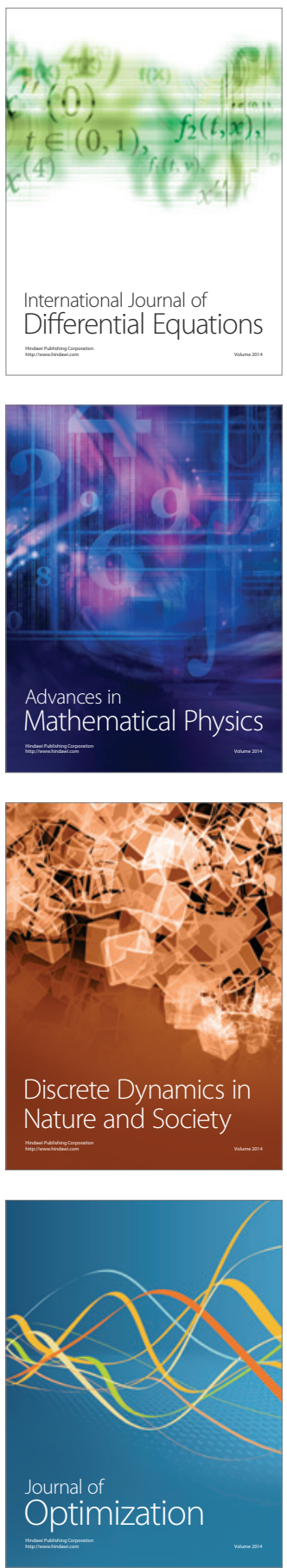